\title{
Rare instances of individuals with Autism Supporting or Engaging in Terrorism: A Reply
}

\author{
Allely, C. S., \& Faccini, L.
}

Dr Clare S Allely. Lecturer in Psychology, School of Health Sciences, University of Salford, Manchester, England and affiliate member of the Gillberg Neuropsychiatry Centre, Sahlgrenska Academy, University of Gothenburg, Gothenburg, Sweden.

Dr Lino Faccini is a Licensed Psychologist based in Hauppauge, New York, USA.

We thank Chown et al for their comments on our paper and we appreciate the opportunity to present our perspective of the need for research within this important field of study despite the controversial and sensitive nature of the topic at hand. As pointed out in the letter, we make it clear to readers in our paper that the involvement of persons with autism spectrum disorder (ASD) in terrorism is a rare occurrence - this is also reflected in our title: 'Rare Instances of Individuals with Autism Supporting or Engaging in Terrorism'. In no way do we support any assertion whatsoever that individuals with ASD are terrorists. As mentioned, we have clearly stated that the vast majority of individuals with ASD are not supporting or engaging in terrorism. However, we may actually be understating the argument because, of course, terrorism seems to be overrepresented in autism. Specifically, terrorism is still extremely rare in the western world and relatively speaking, on the basis of what has been found in the little empirical studies carried out to date, ASD does appears to be present in more than one per cent of the cases (Corner et al., 2015). In Corner, Gill, and Mason's (2016) sample of 153 lone-actor terrorists they found that 3.3\% had ASD. The paper did not state that these three individuals also had co-morbidities and we thank the authors of the letter for obtaining this information directly from the authors privately. It is an important consideration. Nevertheless, Corner and colleagues also stated, on the basis of their studies findings, that: "Three disorders exhibited a higher prevalence in the lone-actor sample than in the general population (schizophrenia, delusional disorder, autism spectrum disorders)". Such important research and findings cannot be ignored. More research is urgently needed to further understand this area.

Chown et al highlight that a number of the cases we discussed in our paper had comorbid intellectual disability (ID) and in one specific case, severe ID. We do recognise that ASD per se is not an ID. We agree that, where there is comorbid ID in addition to ASD, the situation is more complex 
and conclusions cannot be drawn so easily about ASD (or ID). However, why would ID in itself be a more likely candidate to increase the vulnerability in someone to support or engage in terroristic activities/behaviours? We would contend that it would be quite difficult to be involved in lone terrorism only on the basis of low IQ. It is possible that it would be more unlikely than anything else to be specifically linked to acts that require planning and thinking - unless you also have ASD.

Virtually all individuals who actually receive a diagnosis of autism have more than autism (different comorbidities from case to case). This point has been discussed in a number of recent papers. For instance, Gillberg (2010) raised an important clinical consideration that: "Co-existence of disorders including attention-deficit/hyperactivity disorder, oppositional defiant disorder, tic disorder, developmental coordination disorder, and autism spectrum disorder - and sharing of symptoms across disorders (sometimes referred to as comorbidity) is the rule rather than the exception in child psychiatry and developmental medicine". More recently, Gillberg and Fernell (2014) highlighted that the reported prevalence of ASD is increasing. They suggest that a proportion of the increase in the rate of ASD is driven by "Autism Plus". Autism Plus refers to autism with comorbidities (including ID, language disorder, and attention-deficit/hyperactivity disorder or ADHD), and this is what is now being diagnosed by clinicians as ASD. Given the above and the findings, it would take forever or be impossible to ever show if comorbidities such as: bipolar disorder, schizophrenia, epilepsy, intellectual disability (ID), language disorder or whatever other comorbidity actually led the way to terrorism or if it was autism "in itself".

We made a number of points in our paper about why ASD is actually more likely to be "the condition to blame", and we think it would be very difficult to find an argument why bipolar disorder would be specifically "to blame", just to mention one of the comorbidities that the authors bring forth in their letter.

Chown et al regarding the case of Jake Edwards, make the comment that: "Jake was 9 years old, and it is hardly uncommon for children of this age to make comments for effect. We do not regard this as evidence". We are unclear as to what Chown et al are getting at here exactly. Would the diagnosis be wrong just because the individual was nine years? We do not believe so.

Dr Lino Faccini and myself maintain that we have shown the best possible evidence and it is difficult to not conclude that some individuals with ASD might be at high risk of being involved in these terrorist acts. In the letters, the authors state: "Although there are factors that may predispose an autistic individual to be radicalized, there are also factors in autism which would mitigate against 
involvement in any illegal activity". This is exactly the point we are wanting to highlight. That there may exist in some individuals with ASD predisposing factors which may increase the vulnerability to being radicalised. It is exactly what these factors are in individuals with ASD and to what exact they may be involved in such cases that is important to highlight and investigate further. It is only by understanding this through further research that we can begin to understand the potential vulnerabilities in order that preventive and supportive strategies be available and implemented or put in place when necessary. Chown et al state that: "Highlighting autism appears inconsistent with Allely's agreement with Al-Attar that "there is no empirical evidence to show that people on the autism spectrum are at increased risk of engaging in terrorist offences or that autism is overrepresented in terrorist offenders." Given the relatively small number of studies which have shown that ASD may have a higher prevalence in lone-actor terrorist samples (e.g., Corner et al., 2016), clearly there is a need for more research to look at this factor. Might there be cases of individuals with ASD serving sentences for engaging in terroristic behaviours despite having no intention to carry out harm to others? That they were expressing a preoccupation with a topic that may otherwise be considered terroristic behaviours or supporting of terrorism due to their ASD characteristics, for instance? We contend, given anecdotal evidence, that there may well be a number of these cases.

It is the key purpose of our paper in the Journal of Intellectual Disabilities and Offending Behaviour to put into perspective certain suggested findings regarding autism and violence and terrorism (represented in the media, etc), that have been misunderstood, and to refocus the discussion onto what may really be occurring, management of the rare instances and preventing any further stigmatisation. We are well aware and recognise that the majority of individuals with ASD never become involved with the criminal justice system. However, there is a small subgroup who do. It is crucial that we explore the potential vulnerabilities in this small subgroup of individuals with ASD that might put them at greater risk of being drawn into/getting involved in particular crimes or being recruited by others to engage in offending behaviours. One of the key reasons for this is to develop a greater understanding of what these vulnerabilities might be for particular crimes in order that preventive strategies can be put in place to help protect some of these individuals. Current research does not show a direct link but people with autism may be at greater risk of supporting or engaging in terrorism. For those that find themselves faced with such charges, is it imperative that there is research and understanding of how their characteristics of autism may have contributed to their behaviour (the charge that they are faced with). There is increasing anecdotal evidence which has highlighted some of the vulnerabilities in some individuals with ASD which has increased the risk of 
them supporting or engaging in terrorism. Some individuals can be 'manipulated' in to supporting/engaging in terrorism. Despite the sensitive nature of such topics, it is nevertheless crucial that it is further investigated so that papers examining this can be used to ensure a fair trial. This could lead to a number of possibilities in some cases including: reduced sentencing, diversionary measures, or suspended sentences. It is exactly this possibility which prompted in us the need to publish this paper. As pointed out in the letter in response to our paper regarding the case study of Mark Alexander Harding: "It was recognized that Harding had not been radicalized and his online persona was a by-product of his ASD which caused him to develop obsessions over specific subjects". The judge delivering sentence on Harding stated: "It is clear from the report that you suffer from autism and what you did was a form of role play. This is not a case about terrorism this is a case about a 21-year-old who got obsessed with a topic because of his autism." In the letter in response to our paper the readers question whether: "Under these circumstances, is it appropriate for the authors to describe him as a "passive supporter of terrorism?". We believe that, according to law, this individual was passively engaged in support terrorism. It just so happened that in this case, the judge delivering the sentence recognised how his ASD contributed to his behaviour. There is no doubt other similar cases where ASD is not recognised and the individual receives a prison sentence occurs. There is a modest, although important, number of studies which have highlighted the difficulties faced by individuals with ASD in the prison environment. In many cases, prison in not the right place for some individuals with ASD (Allely, 2015). It is cases such as that of Mark Alexander Harding (for example) that we want to highlight and for our paper to be used in the defence in such cases (amongst other types as highlighted in our paper). It is through such research and publications that changes can be made in practice. Whether that be through increased awareness and education amongst criminal justice professionals about how ASD characteristics may contribute to certain offending behaviours or through the use of such papers in psychiatric testimonies, for instance. I have published earlier this year, a paper with my colleague, Professor Penny Cooper on evolving professional responsibilities, liabilities and 'judgecraft' when a party has Asperger's Syndrome (Cooper \& Allely, 2017). This paper is currently being increasingly used throughout the UK and Ireland as the 'go to' when dealing with cases involving clients with ASD. We also have a paper, soon to be published, which highlights the importance of looking at studies which have investigated both juror's and judge's evaluation and perceptions of defendants with ASD and the impact on sentencing thereafter. There is some anecdotal evidence to support the idea that some defendants with ASD are given harsher sentencing due to their clinical presentation in court and the lack of understanding of ASD. 
As emphasised in our paper, there is a very real need for further research in this area and we took the - we believe necessary - step to highlight this need and the importance of considering the presence of ASD in cases involving defendants who find themselves facing charges of terrorism across all levels of terroristic engagement.

\section{References}

Allely, C. S. (2015). Experiences of prison inmates with Autism Spectrum Disorders and the knowledge and understanding of the spectrum amongst prison staff: A review. Journal of Intellectual Disabilities and Offending Behavior, 6(2), 55 - 67.

Allely, C. S., \& Cooper, P. (accepted). A systematic PRISMA review of autism spectrum disorder in the courtroom: Juror's and judge's evaluation of defendants with autism and the impact on sentencing. Journal of Law and Medicine.

Cooper, P., \& Allely, C. S. (2017). You can't judge a book by its cover: evolving professional responsibilities, liabilities and 'judgecraft' when a party has Asperger's Syndrome. Northern Ireland Legal Quarterly, 68(1), 35-58.

Corner, E., Gill, P., \& Mason, O. (2016). Mental health disorders and the terrorist: A research note probing selection effects and disorder prevalence. Studies in Conflict and Terrorism, 39(6), 560-568.

Gillberg, C. (2010). The ESSENCE in child psychiatry: early symptomatic syndromes eliciting neurodevelopmental clinical examinations. Research in Developmental Disabilities, 31(6), 1543-1551.

Gillberg, C., \& Fernell, E. (2014). Autism plus versus autism pure. Journal of Autism and Developmental Disorders, 44(12), 3274-3276. 